\title{
Accuracy of a smartwatch based single-lead electrocardiogram device in detection of atrial fibrillation
}

\author{
Kevin Rajakariar (D) ,' Anoop N Koshy (D) , ${ }^{2}$ Jithin K Sajeev, ${ }^{1}$ Sachin Nair, ${ }^{3}$ \\ Louise Roberts, ${ }^{1,3}$ Andrew W Teh ${ }^{1,3}$
}

${ }^{1}$ Department of Cardiology, Box Hill Hospital, Box Hill, Victoria,

Australia

${ }^{2}$ Department of Cardiology, University of Melbourne, Austin Health, Melbourne, Victoria, Australia

${ }^{3}$ Eastern Health Clinical School Monash University, Clayton, Victoria, Australia

\section{Correspondence to}

Dr Andrew W Teh, Department of Cardiology, Box Hill Hospital, Box Hill, Victoria 3128, Australia; andrew.teh@easternhealth. org.au

KR and ANK are joint first authors.

Received 21 September 2019 Revised 10 December 2019 Accepted 13 December 2019 Published Online First 7 January 2020

\section{ABSTRACT}

Objective The AliveCor KardiaBand (KB) is an Food and Drug Administration-approved smartwatch-based cardiac rhythm monitor that records a lead-Intelligent ECG (iECG). Despite the appeal of wearable integrated ECG devices, there is a paucity of data evaluating their accuracy in diagnosing atrial fibrillation (AF). We evaluated whether a smartwatch-based device for AF detection is an accurate tool for diagnosing AF when compared with 12-lead ECG.

Methods A prospective, multi-centre, validation study was conducted in an inpatient hospital setting. The KB paired with a smartwatch, generated an automated diagnosis of AF or sinus rhythm (SR). This was compared with a 12-lead ECG performed immediately after iECG tracing. Where an unclassified or no-analysis tracing was generated, repeat iECG was performed.

Results 439 ECGs (iECGs ( $n=239)$ and 12-lead ECG $(n=200))$ were recorded in 200 patients (AF: $n=38$; SR: $n=162)$ from three tertiary centres. Sensitivity and specificity using KB was $94.4 \%$ and $81.9 \%$ respectively, with a positive predictive value of $54.8 \%$ and negative predictive value of $98.4 \%$. Agreement between 12-lead ECG and KB diagnosis was moderate when unclassified tracings were included ( $\kappa=0.60,95 \% \mathrm{Cl} 0.47$ to 0.72 ).

Combining the automated device diagnosis with blinded electrophysiologists (EP) interpretation of unclassified tracings improved overall agreement (EP1: $\kappa=0.76$, $95 \% \mathrm{Cl} 0.65$ to 0.87 ; $\mathrm{EP} 2: \mathrm{\kappa}=0.74,95 \% \mathrm{Cl} 0.63$ to 0.86).

Conclusion The KB demonstrated moderate diagnostic accuracy when compared with a 12-lead ECG.

Combining the automated device diagnosis with EP interpretation of unclassified tracings yielded improved accuracy. However, even with future improvements in automated algorithms, physician involvement will likely remain an essential component when exploring the utility of these devices for arrhythmia screening. Clinical trial registration URL: https://www.anzctr. org.au/ Unique identifier: ACTRN12616001374459.

http://dx.doi.org/10.1136/ heartjnl-2019-316390

\section{Check for updates}

(c) Author(s) (or their employer(s)) 2020. No commercial re-use. See rights and permissions. Published by BMJ.

To cite: Rajakariar K, Koshy AN, Sajeev JK, et al. Heart 2020;106:665-670.

\section{INTRODUCTION}

Wearable arrhythmia detection devices are becoming increasingly popular, with sales set to double by $2022 .{ }^{1-4}$ These devices obtain physiological data using a simple user interface to instantaneously provide a diagnosis and transmit information to a receiving cardiologist for prompt review. With consumer interest driving the rapid expansion of portable healthcare, this ultimately culminated in the US Food and Drug Administration (FDA) clearing several technologies including the AliveCor KardiaBand (KB), which is a strap for the Apple watch (Apple, Cupertino, California, USA) with an inbuilt electrode that generates a leadIntelligent ECG (iECG) and provides an automated diagnosis of either atrial fibrillation (AF) or sinus rhythm (SR). If proven to be accurate, these devices have the potential to be used for population-level screening of $\mathrm{AF}$ with subsequent prescription of oral anticoagulation to prevent stroke. However, before population screening can be effective, the accuracy of the diagnostic test requires rigorous validation. While there is high clinician interest and consumer expectation of diagnostic precision from these devices, there is a paucity of data evaluating their accuracy in arrhythmia diagnosis. ${ }^{5}$ In addition, although such devices are freely available to the consumer, there is insufficient data from clinical trials to guide clinicians on how to interpret their data and integrate it into the decisionmaking process. We aimed to evaluate the accuracy of the $\mathrm{KB}$ as a detection tool for $\mathrm{AF}$ in an unselected hospital cohort.

\section{METHODS}

A prospective, multi-centre validation study was performed across three tertiary university hospitals, where consecutive patients $\geq 18$ years of age admitted to the medical, cardiac or intensive care ward were invited to participate. Patients with cardiac implantable electronic devices, those unable to independently use the device, or in contact isolation were excluded from the study. Written informed consent was obtained from all subjects. Baseline characteristics including demographic data, cardiac risk factors and medications were obtained.

The KB strap was attached to an Apple Watch and paired with an iPhone 6 smartphone (Apple, Cupertino, California, USA) using the AliveCor Kardia application V.5.0.2 (AliveCor, Mountain View, California, USA). The device obtains a 30 -second continuous lead-I recording that can be viewed in real-time on the iPhone and is remotely transmitted to a secure server (US Health Insurance Portability and Accountability standards compliant) for storage and subsequent clinician analysis. The KB analysis then displays a diagnosis of either (a) possible AF; 


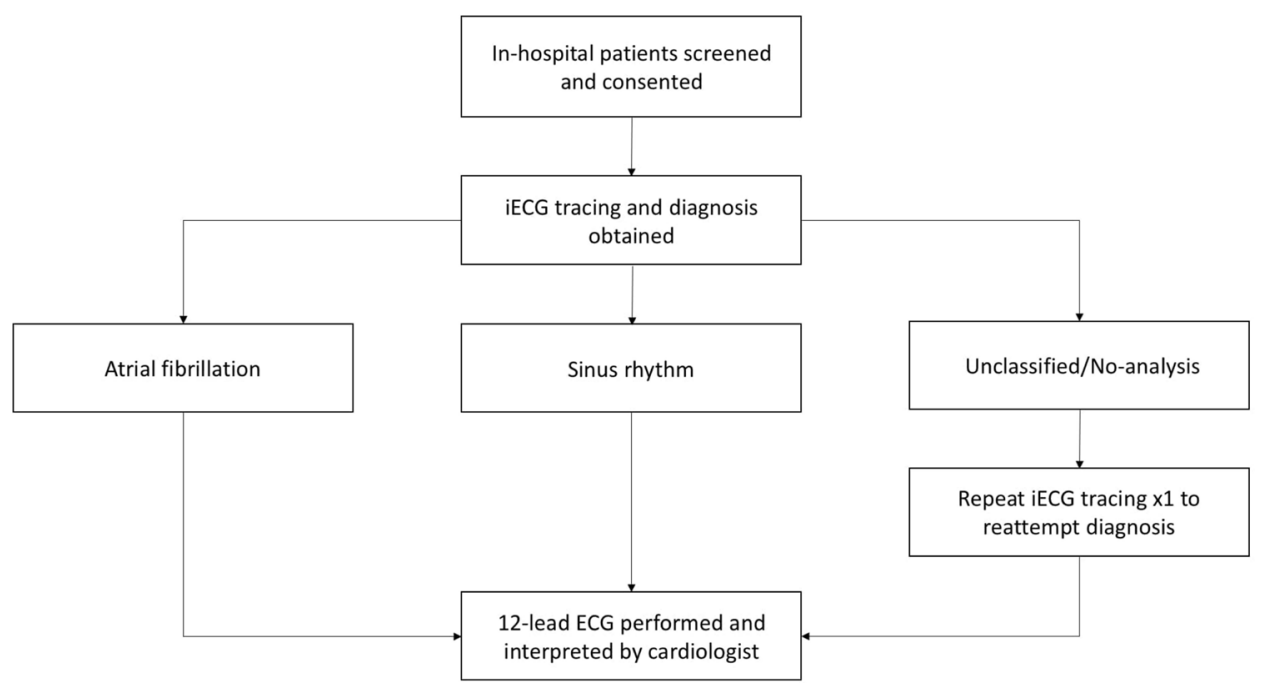

Figure 1 Study protocol. EECG, intelligent ECG.

(b) normal SR; (c) no-analysis or (d) unclassified. The no-analysis reading is displayed when there is excessive artefact or interference for the $\mathrm{KB}$ algorithm to determine a diagnosis. An unclassified reading is shown when, in the absence of recording artefact, the device is unable to determine whether the trace is $\mathrm{AF}$ or SR. Bradycardia was defined as heart rate $<50$ beats per minute (bpm) and tachycardia $\geq 100 \mathrm{bpm}$, and when detected resulted in an unclassified reading. An ECG trace was generated by wearing the Apple Watch on the wrist, and placing the thumb of the opposite hand onto the electrode located on the KB strap for 30 seconds. Where an unclassified or no-analysis reading was displayed, a second $\mathrm{KB}$ tracing was obtained and used as the final diagnosis (figure 1). All patients were supervised and instructed in a standardised manner, and the initial recorded iECG was used with no test recordings performed prior.

Patients were screened for inclusion on the wards. After recruitment, patients were consented and an iECG tracing was obtained. A 12-lead ECG was performed immediately following the $\mathrm{KB}$ trace, for assessment of $\mathrm{KB}$ accuracy and to minimise the chance of rhythm variance between tracings. There were no opportunities for patients to perform additional iECG tracings. $\mathrm{KB}$ tracings were only obtained during initial recruitment and were not taken during symptomatic episodes or at any alternative time. The ECG was interpreted and diagnosis subsequently determined by a cardiologist (ANK) and used as the reference standard against the KB device. The ECG automated diagnosis was not used. Sensitivity, specificity, predictive value, likelihood ratios were calculated using $2 \times 2$ contingency tables. Accuracy (A) was estimated by determining the percentage of true positive (TP) and true negative (TN) results compared with all results (R) defined as $A=(T P+T N) \div R$. When analysing KB accuracy by assessing unclassified diagnoses as incorrect, AF was categorised as a false negative and SR as a false positive. Although some arrhythmias were correctly given an unclassified diagnosis, they were considered incorrect to conservatively measure KB accuracy and in a real-world scenario, all unclassified diagnoses would require clinician interpretation. All unclassified tracings were compiled for diagnostic assessment by two independent blinded cardiac electrophysiologists (EP1: JKS, EP2: AWT). Due to the artefactual nature of no-analysis readings, consecutive iECGs generating this diagnosis were not included in the statistical analysis. Overall agreement between the KB, electrophysiologists, and 12-lead ECG was analysed using Cohen's kappa coefficient ( $\kappa)$. All analyses were two-tailed, and $\mathrm{p}$ values of $<0.05$ were considered statistically significant. Statistical analyses were performed using Stata V.13/MP.

\section{Patient and public involvement statement}

The patients and public were not involved in the creation of the study design, recruitment or statistical analysis. Patients were not consulted to develop patient relevant outcomes or interpret the results. Patients were not invited to contribute to the writing or editing of this document for readability or accuracy.

\section{RESULTS}

A total of 218 consecutive patients were recruited over 6 months, with 200 patients $(56.5 \%$ male, age $67 \pm 16$ years) eligible for inclusion into the study. Overall, 439 ECGs were recorded (iECGs $(n=239)$ and 12-lead ECG $(n=200))$. This included 38 (19\%) patients in AF, and 162 in SR based on the interpreted 12-lead ECG diagnosis (determined by ANK). Eighteen patients were excluded as they did not fulfil inclusion criteria (implanted cardiac device $(n=8)$, significant tremor $(n=6)$, contact isolation $(\mathrm{n}=4))$. The baseline clinical characteristics are summarised in table 1. Patients with AF were more likely to be older with more comorbid conditions.

A summary of the iECG results is shown in figure 2. Overall, $161(80.5 \%) \mathrm{AF}$ and SR diagnoses were obtained from the initial iECG (figures 3 and 4). Fourteen tracings (7\%) were recorded as no-analysis and $25(12.5 \%)$ as unclassified readings (figures 5 and 6). A second iECG performed $(n=39)$ recorded a diagnosis in 14 patients $(7 \%)$ with the remainder being unclassified $(8 \%)$ or no-analysis (4.5\%). In total, 175 (87.5\%) AF and SR diagnoses were obtained using up to two iECGs from the patient cohort. On review of unclassified diagnoses, seven (3.5\%) were given the diagnosis in the setting of sinus tachycardia, three $(1.5 \%)$ contained premature atrial complexes, three $(1.5 \%)$ were sinus rhythm with first degree atrioventricular block, and three (1.5\%) iECGs had unclear reasons for remaining unclassified. No tracings were given an unclassified diagnosis due to bradycardia. All no-analysis tracings were in the setting of artefact (figure 7), including two (1\%) patients unable to complete the tracing due to Parkinsonian tremor.

The iECG automated algorithm demonstrated an overall sensitivity of $94.4 \%$ when categorising unclassified readings as incorrect, and improved to $95.4 \%$ when incorporating tracings which 


\begin{tabular}{|c|c|c|c|}
\hline & $\begin{array}{l}\text { Normal sinus } \\
\text { rhythm ( } n=162)\end{array}$ & $\begin{array}{l}\text { Atrial } \\
\text { fibrillation } \\
(\mathrm{n}=38)\end{array}$ & $P$ value \\
\hline Age in years $($ mean $\pm S D)$ & $64 \pm 17$ & $76 \pm 11$ & $<0.001$ \\
\hline Male & $64 \%$ & $52 \%$ & 0.64 \\
\hline Ischaemic heart disease & $32 \%$ & $50 \%$ & 0.09 \\
\hline Hypertension & $51 \%$ & $50 \%$ & 0.36 \\
\hline Heart failure & $13 \%$ & $44 \%$ & $<0.001$ \\
\hline Diabetes & $20 \%$ & $25 \%$ & 0.53 \\
\hline Stroke/TIA & $16 \%$ & $17 \%$ & 0.93 \\
\hline Peripheral vascular disease & $10 \%$ & $14 \%$ & 0.07 \\
\hline Known atrial flutter/fibrillation & $9 \%$ & $95 \%$ & $<0.001$ \\
\hline History of palpitations & $26 \%$ & $79 \%$ & $<0.001$ \\
\hline Mean $\mathrm{CHA}_{2} \mathrm{DS}_{2}$ VASc score & 2 & 3 & 0.02 \\
\hline Antiarrhythmic medications (any) & $6 \%$ & $44 \%$ & 0.001 \\
\hline \multicolumn{4}{|l|}{ Antiarrhythmic medications } \\
\hline Flecainide & $1 \%$ & $0 \%$ & \\
\hline Beta-blocker & $22 \%$ & $100 \%$ & \\
\hline Amiodarone/sotalol & $5 \%$ & $14 \%$ & \\
\hline Calcium channel blocker & $1 \%$ & $0 \%$ & \\
\hline Digoxin & $1 \%$ & $14 \%$ & \\
\hline \multicolumn{4}{|l|}{ Anticoagulation } \\
\hline Warfarin & $0 \%$ & $0 \%$ & \\
\hline Apixaban & $4 \%$ & $57 \%$ & \\
\hline Rivaroxaban & $4 \%$ & $21 \%$ & \\
\hline Dabigatran & $0 \%$ & $14 \%$ & \\
\hline Heparin & $0 \%$ & $7 \%$ & \\
\hline
\end{tabular}

$P$ values $<0.05$ demonstrate a significant difference between patients with normal sinus rhythm and atrial fibrillation. Patients in atrial fibrillation were older, with a higher incidence of palpitations and heart failure.

TIA, transient ischaemic attack.

were appropriately given an unclassified diagnosis (due to sinus tachycardia). When these readings were excluded, the sensitivity remained at $94.4 \%$ as all repeat iECG unclassified readings were

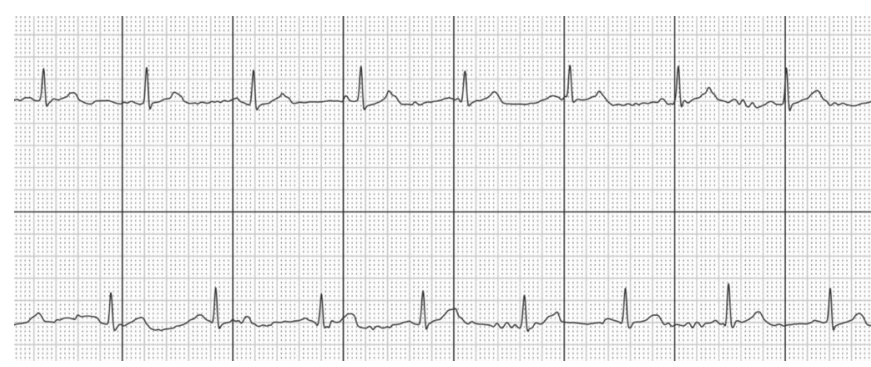

Figure 3 iECG tracing of sinus rhythm. iECG, intelligent ECG.

SR. The KB specificity was $81.9 \%$, which increased to $90.7 \%$ when excluding unclassified readings due to a reduction in false positive diagnoses. The positive and negative predictive values for the KB (excluding unclassified diagnoses) were $72.3 \%$ and $98.4 \%$ respectively. Agreement between the single lead KB diagnosis and 12-lead ECG cardiologist diagnosis when including unclassified tracings (marked as incorrect) was moderate $(\kappa=0.60,95 \% \mathrm{CI}$ 0.47 to $0.72, \mathrm{p}<0.001$ ), however improved when incorporating appropriately diagnosed unclassified readings $(\kappa=0.71,95 \% \mathrm{CI}$ 0.62 to $0.77, \mathrm{p}<0.001)$ and excluding unclassified readings altogether $(\kappa=0.76,95 \%$ CI 0.67 to $0.88, \mathrm{p}<0.001)$.

Review of unclassified tracings by two electrophysiologists blinded to the 12-lead ECG diagnosis revealed an overall accuracy of 93.8\% (EP1: 1 false positive, 0 false negatives) and $87.5 \%$ (EP2: 2 false positives, 0 false negatives) respectively. When incorporating EP diagnoses of unclassified readings with the KB automated analysis, we demonstrate improved positive predictive value (70.8\% and 69.4\% for EP1 and EP2 respectively) and improved agreement with the 12-lead ECG cardiologist diagnosis (EP1: $\kappa=0.76,95 \% \mathrm{CI} 0.65$ to $0.87, \mathrm{p}<0.001$; EP2: $\kappa=0.74,95 \% \mathrm{CI}$ 0.63 to $0.86, \mathrm{p}<0.001)$. Interobserver agreement between EPs was strong $(\kappa=0.98,95 \%$ CI 0.91 to $1.00, \mathrm{p}<0.001)$. A summary of results with clinician interpretation is outlined in table 2 .

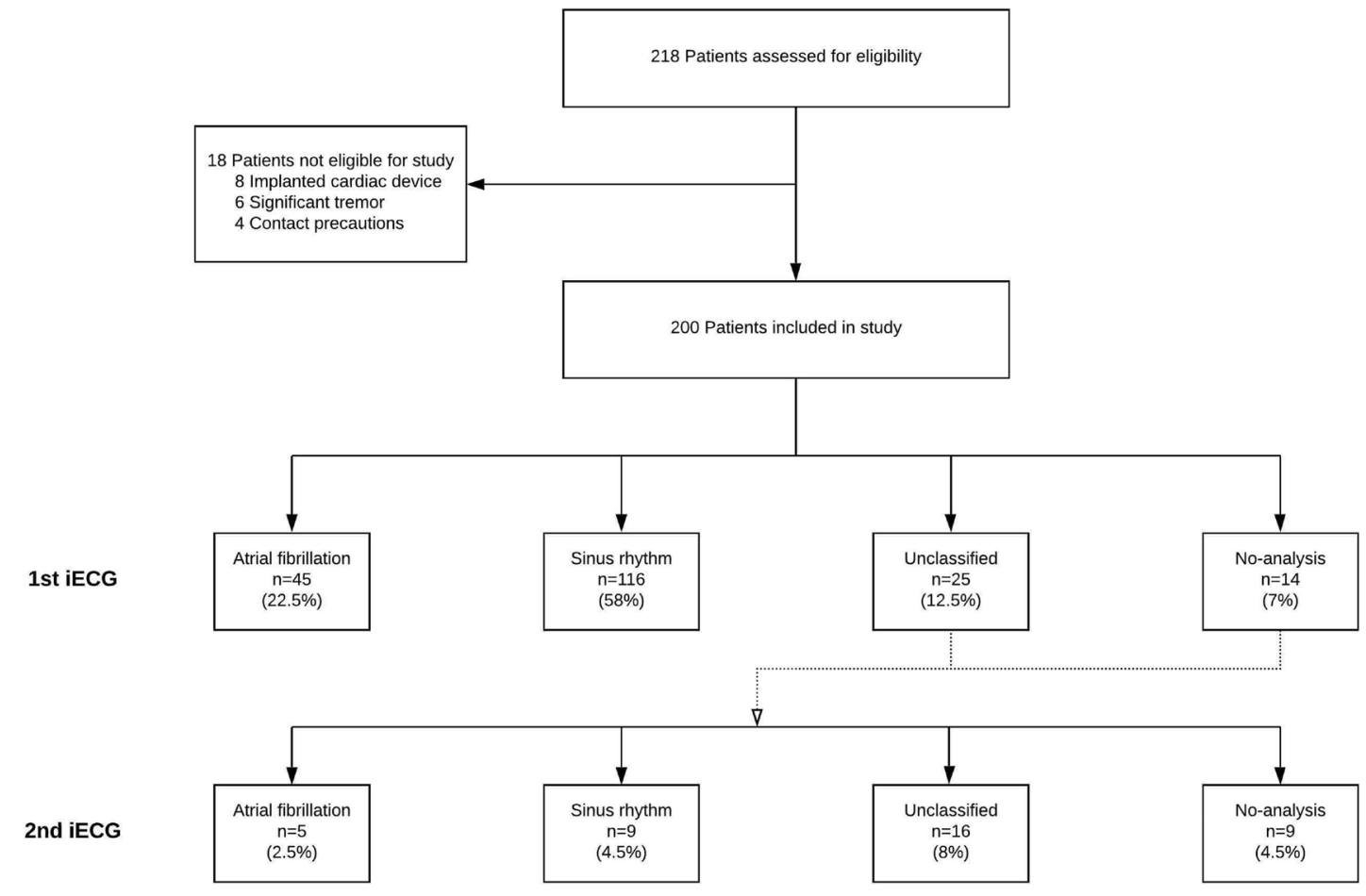

Figure 2 Summary of iECG results—a total of $39(19.5 \%)$ iECGs were uninterpretable and subsequently repeated, of which 14 (7\%) generated a diagnosis. iECG, intelligent ECG. 


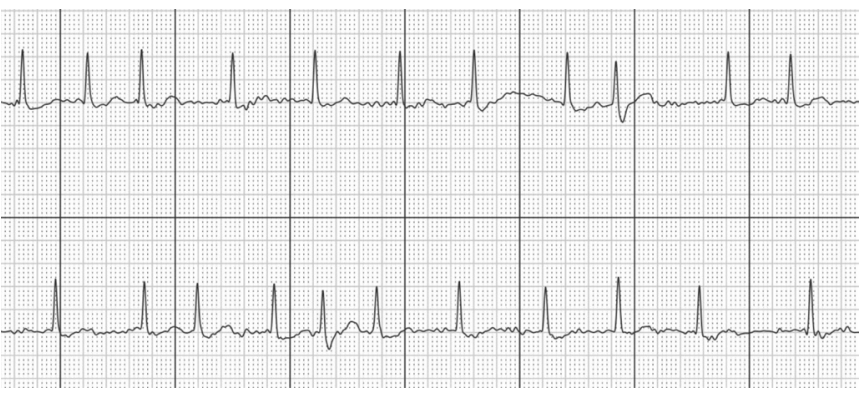

Figure 4 iECG tracing of atrial fibrillation. iECG, intelligent ECG.

The majority of patients with AF had a pre-existing history of the condition. However, there were two new diagnoses of AF, confirmed on both 12-lead ECG cardiologist interpretation and iECG automated diagnosis. Both patients described a history of palpitations, but were asymptomatic during the time of the AF recordings.

\section{DISCUSSION}

This study evaluated the accuracy of a novel smartwatch-based ECG monitor for diagnosing AF in an unselected high-risk inpatient population. When a KB automated diagnosis was proffered, it demonstrated high sensitivity for diagnosing AF. A significant number of false positives were noted for both device automated diagnosis of AF and clinician interpretation of unclassified readings. Although overall $\mathrm{KB}$ accuracy was moderate when categorising unclassified tracings as incorrect, incorporating the $\mathrm{KB}$ automated diagnosis with EP interpretation of only unclassified tracings yielded improved diagnostic accuracy.

The initial iECG also demonstrated a $19.5 \%$ rate of undiagnosed tracings, which nearly halved once a repeat iECG was performed, consistent with similar studies using AliveCor technology. ${ }^{67}$ The exact reasons for the relatively high rates of no analysis and unclassified tracings is unclear. However, recruitment of older, hospitalised patients could have affected the ability for patients to record the tracings without generation of artefact that may have altered the accuracy of the device algorithm (figure 5). While many of these tracings were due to electrical noise during the period of iECG acquisition, some of our unclassified diagnoses were appropriate in certain non-AF arrhythmias such as sinus bradycardia or tachycardia (figure 6). Such rhythms are more likely to occur in a hospitalised cohort and therefore cautious interpretation is required when analysing the device rate of unclassified diagnoses. In addition, where an unclassified or no-analysis diagnosis is specified, a repeat iECG

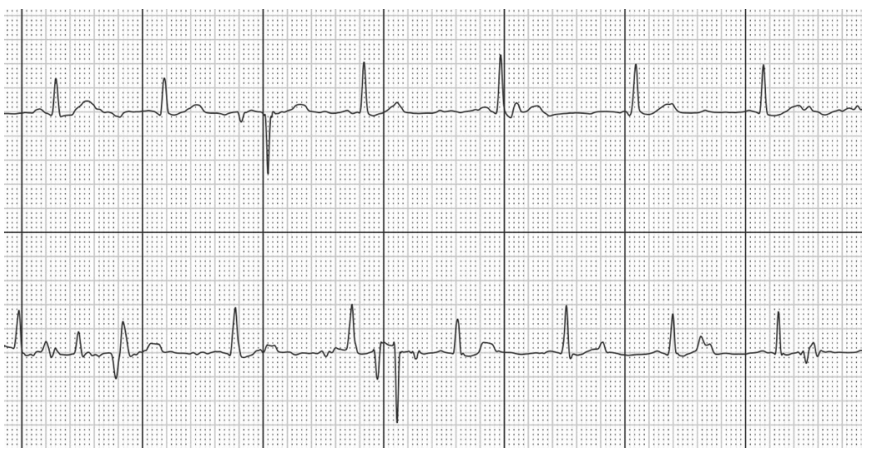

Figure 5 iECG tracing of an unclassified rhythm. Clinician over-read correctly identified this as sinus rhythm. iECG, intelligent ECG.

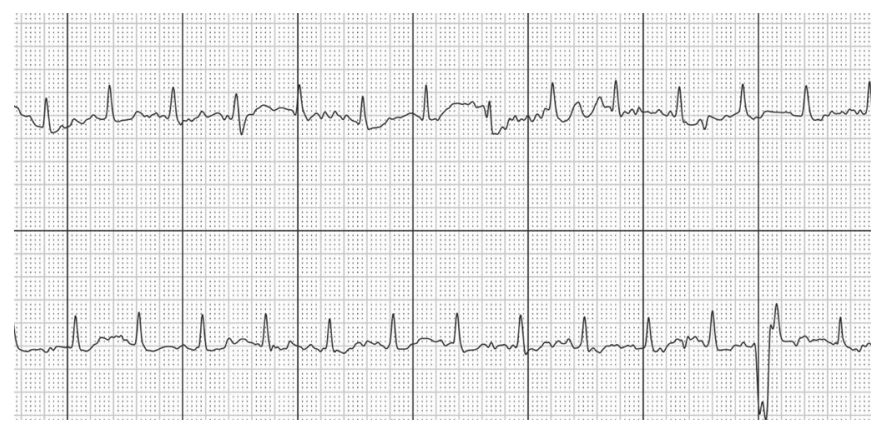

Figure 6 iECG tracing of an unclassified rhythm, in the setting of tachycardia. iECG, intelligent ECG.

should be performed to help differentiate from artefactual causes for the given diagnosis.

There is increasing interest in the use of smartphone-based technology in AF detection with numerous published feasibility and screening studies. ${ }^{8-10}$ However, multiple thorough validation studies are required to confirm their accuracy before they can be recommended for population level use, with the ultimate goal being accurate early detection of AF leading to prevention of stroke. Since the recent FDA approval and release of the smartwatch-based ECG devices, only two previous studies have reported on its accuracy. However, both studies experienced high dropout rates, with one trial conducted in a preselected patient cohort undergoing cardioversion, limiting its generalisability. ${ }^{711}$ This is particularly pertinent given that pretest probability of AF significantly affects testing parameters with prior studies reporting a positive predictive value as low as 5\% for AF in an unselected patient population. ${ }^{12-14}$ In our study, in an unselected hospital patient population, the KB algorithm yielded high sensitivity with a significant number of unclassified readings; this would be expected to improve over time with rapid developments in AF detection algorithms including the use of deep neural networks. ${ }^{15}$ In addition, given the significant proportion of false positives, using a diagnosis of AF from the device to guide commencement of treatment such as oral anticoagulants for stroke prophylaxis cannot be recommended at this stage. With increasing adoption of wearable arrhythmia devices, establishing the accuracy of these technologies is crucial and needs to occur before clinicians can begin to even consider whether subsequent investigations and therapies should be instituted.

With rapid developments in arrhythmia detection technology, there are numerous advantages of smartwatch linked devices compared with their smartphone counterparts. Improved accessibility of smartwatch-based devices allows the consumer the

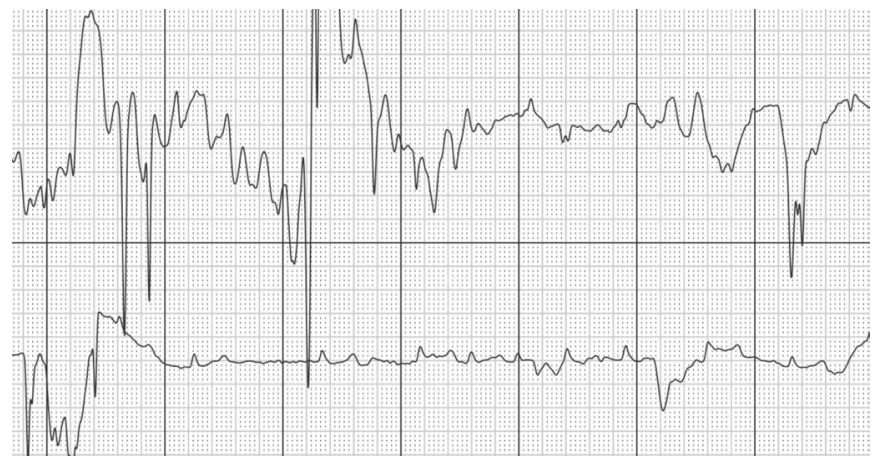

Figure 7 iECG tracing of a no-analysis rhythm, likely due to significant artefact. 
Table 2 Diagnosing AF with and without clinician over-read of unclassified readings, against ECG as gold standard

\begin{tabular}{|c|c|c|c|c|c|c|c|c|c|c|}
\hline KardiaBand & Sensitivity (\%) & Specificity (\%) & PPV (\%) & NPV (\%) & PLR & NLR & Accuracy (\%) & Kappa & $95 \% \mathrm{Cl}$ & $P$ value \\
\hline Consecutive unclassified excluded & 94.4 & 90.7 & 72.3 & 98.4 & 10.2 & 0.06 & 91.5 & 0.76 & 0.67 to 0.88 & $<0.001$ \\
\hline Consecutive unclassified marked as incorrect & 94.4 & 81.9 & 54.8 & 98.4 & 5.2 & 0.07 & 84.3 & 0.60 & 0.47 to 0.72 & $<0.001$ \\
\hline $\mathrm{KB}+$ appropriate unclassified diagnosis & 95.4 & 81.9 & 59.4 & 98.5 & 5.2 & 0.06 & 84.9 & 0.71 & 0.62 to 0.77 & $<0.001$ \\
\hline $\mathrm{KB}+\mathrm{EP} 1$ review (only unclassified) & 94.4 & 91.0 & 70.8 & 98.6 & 10.5 & 0.06 & 91.7 & 0.76 & 0.65 to 0.87 & $<0.001$ \\
\hline $\mathrm{KB}+\mathrm{EP} 2$ review (only unclassified) & 94.4 & 90.4 & 69.4 & 98.6 & 9.8 & 0.06 & 91.2 & 0.74 & 0.63 to 0.86 & $<0.001$ \\
\hline
\end{tabular}

Detailed analysis of KB accuracy for diagnosing AF with and without clinician interpretation of unclassified tracings, against cardiologist ECG diagnosis as gold standard. When the initial iECG gave an unclassified reading, a repeat iECG was taken and used as the final diagnosis. An appropriate unclassified diagnosis was determined if the tracing was due to a non-AF arrhythmia. P values are derived for the kappa coefficient.

AF, atrial fibrillation; EP, electrophysiologist;iECG, intelligent ECG; KB, KardiaBand; NLR, negative likelihood ratio; NPV, negative predictive value; PLR, positive likelihood ratio; PPV, positive predictive value.

ability to perform tracings throughout the day ensuring greater opportunities for detection of paroxysmal AF. Moreover, the incorporation of AliveCor SmartRhythm technology into the $\mathrm{KB}$ uses inbuilt smartwatch photoplethysmography to provide an opportunity for continuous cardiac monitoring. This technology employs a deep neural network to correlate acute fluctuations in heart rate with physical activity, and recent studies have demonstrated excellent accuracy compared with other wearable devices. ${ }^{16}$ Any discordance between the two will prompt the user to acquire an ECG. ${ }^{17}$ Similar technology has been incorporated to the newly released Apple smartwatches, however the accuracy of these technologies has not been published. AliveCor has subsequently released the KardiaMobile 6L, an arrhythmia detection device with the ability to create a hand-held 6-lead iECG using three electrodes. This technology may be complementary to the validation studies performed using $\mathrm{KB}$ with a potential for providing more detailed electrocardiographic data. With further clinical validation studies of these newer devices, clinicians will be better equipped to understand how to incorporate data from these technologies into their practice.

A chief concern highlighted with AF detection platforms are the associated costs involved with clinician interpretation of iECG tracings. In the largest community study using the AliveCor Kardia monitor, the cost for a new diagnosis of AF exceeded $\$ 10,000$, largely driven by expenditure associated with commercial over-reads. ${ }^{12} 13$ Importantly, consumers are not limited in the amount of KB tracings which can be performed, which could lead to increased false positive diagnoses, unclassified readings and clinicians being overwhelmed by the volume of data requiring oversight. In a pilot study, we proposed a pathway whereby limiting clinician interpretation of iECG tracings to unclassified readings could be a more economically viable strategy for arrhythmia screening when using smartphonebased ECG technology. ${ }^{18} 19$ In the present study, incorporating the $\mathrm{KB}$ automated diagnosis with EP interpretation of unclassified tracings alone demonstrated improved accuracy, which is comparable with results of previous studies employing clinician interpretation of all tracings. ${ }^{20} 21$ In a high-risk population, combining KB diagnoses with clinician over-read of unclassified tracings alone may provide a more cost-effective solution than clinician interpretation of all iECG tracings. ${ }^{10}$

Our proposed work-flow of limiting clinician traces to 'unclassified' traces could be challenging in the real-world. Ongoing consumer engagement with these devices may generate increasing numbers of these traces that require clinician overread. Notwithstanding, this may be more feasible than manual over-read of all ECG tracings. Our findings shed light on the feasibility and accuracy of smartwatch-based arrhythmia detection in a hospitalised cohort. However, large scale cohort studies are needed to confirm our findings and the cost-effectiveness of this strategy in an all-comer community-dwelling population. Collaboration among key stakeholders including industry, clinicians and consumers is needed to generate actionable health policy that can leverage the use of wearable device-generated ECG data in improving care provision.

\section{Implications}

Wearables can facilitate affordable large-scale arrhythmia screening at a population level. However, there are several barriers to overcome if such technology is to become the contemporary standard in arrhythmia detection. Current iterations of single-lead iECG devices are heavily reliant on symptoms of palpitations to trigger opportunistic screening, and consequently can overlook patients with paroxysmal arrhythmias. ${ }^{22}$ While alternative wearables using photoplethysmography (PPG) can provide continuous screening, accuracy is currently affected by motion artefact, ectopic beats, peripheral vascular disease, poor skin contact and limited battery life. ${ }^{23}$ In addition, the potential for continuous monitoring is limited by the battery life with current device iterations requiring daily overnight charging. A simplistic user interface will also be required to ensure those at the highest risk of AF-the elderly-will be able to adapt to such technology to not only obtain iECGs, but to interpret its findings and identify when further clinician input is required. Newer devices that can combine PPG with accelerometer data, superior battery life and smarter arrhythmia detection algorithms may overcome the current constraints that limit wearables from becoming the next affordable frontier in arrhythmia screening. With increasing uptake of wearable devices with ECG capabilities, there will be an exponential rise in positive iECG readings. This has potential to culminate in both user anxiety and consumption of valuable health resources for downstream diagnostic testing. ${ }^{19}$ Therefore, algorithms designed to streamline clinician over-read of tracings are also required if population level arrhythmia screening is to occur. Finally, long-term outcomes for patients treated for subclinical or device-detected $\mathrm{AF}$ is unknown; further studies are required to determine the risk of thromboembolic stroke in this subgroup and the efficacy of treatment with oral anticoagulation. ${ }^{24}$

We acknowledge certain limitations of this study. First, our cohort had an AF prevalence rate of $19 \%$, which is significantly higher than the 2\%-5\% population estimates of $\mathrm{AF}^{25} \mathrm{~A}$ higher prevalence of AF in our population could overestimate the calculated predictive values in our study. As such, it would be essential to validate these results in an all-comer outpatient population with a lower pretest probability of AF. Second, it remains unclear whether the frequency of unclassified and uninterpretable tracings is likely to be higher when the device is used at home by patients who may lack the knowledge and training to amend their technique to improve iECG acquisition. This, in turn, could 
increase both unnecessary patient anxiety and the number of iECGs that necessitate clinician review. In our study, all patients were supervised and provided instructions on technique, which is unlikely to occur in a real-world scenario. Furthermore, even if the $\mathrm{KB}$ is established as an accurate tool for diagnosing $\mathrm{AF}$, the threshold at which to treat $\mathrm{AF}$ in otherwise asymptomatic individuals remains unknown. Lastly, only two EPs assessed iECGs in this study. It remains unclear if these findings are generalisable to other physicians and whether prior clinician experience with iECGs affects accuracy.

\section{CONCLUSION}

In an unselected hospital patient cohort, the KB demonstrated moderate diagnostic accuracy when compared with a 12-lead ECG, which improved when uninterpretable traces were excluded. Combining the automated device diagnosis with EP interpretation of unclassified tracings improved both positive predictive value and overall accuracy. However, even with future improvements in automated algorithms, this suggests that physician involvement will likely remain an essential component when exploring the utility of these devices for arrhythmia screening.

\section{Key messages}

What is already known on this subject?

- Wearable arrhythmia detection devices are becoming increasingly popular, with sales set to double in the next 3 years.

- Despite the growing uptake of wearables in the community, there is a paucity of data evaluating the accuracy of these devices.

\section{What might this study add?}

- In a prospective study of 200 patients, the KardiaBand demonstrated moderate diagnostic accuracy compared with a 12-lead ECG when unclassified traces were included.

\section{How might this impact on clinical practice?}

- Combining the automated device diagnosis with clinician interpretation of unclassified tracings yielded improved accuracy.

- Even with future improvements in automated algorithms, physician involvement will likely remain an essential component when exploring the utility of these devices for arrhythmia screening.

\section{Twitter Anoop N Koshy @DrAnoop_Koshy}

Acknowledgements We thank the physicians, nurses and patients involved in the study.

Contributors KR and ANK equally contributed to the initial manuscript draft, recruitment and data analysis. SN was involved in data collection. JKS and LR contributed to data analysis and draft revision. AWT reviewed the data, edited and approved the final submitted draft.

Funding This work was supported by the Eastern Health Foundation Research Grant [EHFRG2017_029]. The sponsor had no role in study design, collection, analysis, interpretation of data and in the decision to submit the article for publication. Dr. Koshy is supported by scholarships from the National Health and Medical Research Council of Australia, National Heart Foundation and The Royal Australasian College of Physicians.

Competing interests None declared.

Patient consent for publication Not required.
Ethics approval Institutional ethics review board approval of the study protocol (LR55-2016).

Provenance and peer review Not commissioned; externally peer reviewed.

Data availability statement All data relevant to the study are included in the article or uploaded as supplementary information. Data are available upon reasonable request

\section{ORCID iDs}

Kevin Rajakariar http://orcid.org/0000-0002-3886-8214

Anoop N Koshy http://orcid.org/0000-0002-8741-8631

\section{REFERENCES}

1 Page T. A forecast of the adoption of wearable technology. Int J Technology Diffusion 2015;6:12-29.

2 Jovin I. Wearables market to double by 2022, Apple Watch expected to fuel growth. [online] Gadgets \& Wearables. Available: https://gadgetsandwearables.com/2018/10/ 24/css-insight-wearables [Accessed Sep 2019].

3 Cheung CC, Krahn AD, Andrade JG. The emerging role of wearable technologies in detection of arrhythmia. Can J Cardiol 2018:34:1083-7.

4 Piwek L, Ellis DA, Andrews S, et al. The rise of consumer health wearables: promises and barriers. PLOS Med 2016:13:e1001953.

5 Ip JE. Wearable devices for cardiac rhythm diagnosis and management. JAMA 2019:321:337-8

6 William AD, Kanbour M, Callahan T, et al. Assessing the accuracy of an automated atrial fibrillation detection algorithm using smartphone technology: the IREAD study. Heart Rhythm 2018;15:1561-5.

7 Bumgarner JM, Lambert CT, Hussein AA, et al. Automated atrial fibrillation detection algorithm using smartwatch technology. J Am Coll Cardiol 2018;71.

8 Rozen G, Vaid J, Hosseini SM, et al. Diagnostic accuracy of a novel mobile phone application for the detection and monitoring of atrial fibrillation. Am J Cardiol 2018;121:1187-91.

9 Tarakji KG, Wazni OM, Callahan T, et al. Using a novel wireless system for monitoring patients after the atrial fibrillation ablation procedure: the iTransmit study. Heart Rhythm 2015;12:554-9.

10 Orchard J, Lowres N, Freedman SB, et al. Screening for atrial fibrillation during influenza vaccinations by primary care nurses using a smartphone electrocardiograph (iECG): a feasibility study. Eur J Prev Cardiol 2016;23:13-20.

11 Dörr M, Nohturfft V, Brasier N, et al. The WATCH AF Trial: SmartWATCHes for Detection of Atrial Fibrillation. JACC Clin Electrophysiol 2019;5:199-208.

12 Halcox JPJ, Wareham K, Cardew A, et al. Assessment of remote heart rhythm sampling using the AliveCor heart monitor to screen for atrial fibrillation. Circulation 2017;136:1784-94.

13 Lowres N, Neubeck L, Salkeld G, et al. Feasibility and cost-effectiveness of stroke prevention through community screening for atrial fibrillation using iPhone ECG in pharmacies. Thromb Haemost 2014;111:1167-76.

14 Koshy AN, Sajeev JK, Teh AW, et al. Letter by Koshy et al Regarding Article, "Assessment of Remote Heart Rhythm Sampling Using the AliveCor Heart Monitor to Screen for Atrial Fibrillation: The REHEARSE-AF Study". Circulation 2018;137:2191-2.

15 Tison GH, Sanchez JM, Ballinger B, et al. Passive detection of atrial fibrillation using a commercially available smartwatch. JAMA Cardiol 2018;3:409-16.

16 Koshy AN, Sajeev JK, Nerlekar N, et al. Smart watches for heart rate assessment in atrial arrhythmias. Int J Cardiol 2018:266:124-7.

17 AliveCor Inc. User manual for Kardia mobile by AliveCor, 2017. Available: https:// www.alivecor.com/previous-labeling/kardiamobile/00LB17.4.pdf [Accessed Sep 2019].

18 Koshy AN, Sajeev JK, Negishi K, et al. Accuracy of blinded clinician interpretation of single-lead smartphone electrocardiograms and a proposed clinical workflow. Am Heart J 2018:205:149-53.

19 Sajeev JK, Koshy AN, Teh AW. Wearable devices for cardiac arrhythmia detection: a new contender? Intern Med J 2019:49:570-3.

20 Lau JK, Lowres N, Neubeck L, et al. iPhone ECG application for community screening to detect silent atrial fibrillation: a novel technology to prevent stroke. Int I Cardiol 2013:165:193-4.

21 Desteghe L, Raymaekers Z, Lutin M, et al. Performance of handheld electrocardiogram devices to detect atrial fibrillation in a cardiology and geriatric ward setting. Europace 2017:19:29-39.

22 Bennett $R$, French A. Rise of the smart device ECG and what it means for the general cardiologist. Heart 2019;105:1763-4.

23 Ip JE. Evaluation of cardiac rhythm abnormalities from wearable devices. JAMA 2019:321:1098-9.

24 Healey JS, Wong J. Wearable and implantable diagnostic monitors in early assessment of atrial tachyarrhythmia burden. Europace 2019;21:377-82.

25 Wong CX, Brown A, Tse H-F, et al. Epidemiology of atrial fibrillation: the Australian and Asia-Pacific perspective. Heart Lung Circ 2017:26:870-9. 